\title{
Desafíos del emprendimiento universitario en Méjico frente a los Objetivos de Desarrollo Sostenible.
}

\author{
Challenges of university entrepreneurship in Mexico \\ ahead of the Sustainable Development Goals
}

\section{María del Pilar Pérez-Hernández - Daniel Akenaton Granillo Hernández MÉJICO}

Resumen: El emprendimiento universitario emerge como una estrategia para el desarrollo económico y social, que configura una senda para impulsar la generación de empleos, innovación y el crecimiento económico sostenible. Los retos que implica la sostenibilidad, plasmados en los Objetivos de Desarrollo Sostenible (ODS), simbolizan áreas de oportunidad para emprendedores. El artículo identifica los desafíos del emprendimiento universitario frente a los ODS. Para ello, primero se hace una revisión de la literatura sobre el emprendimiento, se caracteriza el emprendimiento en Méjico y finalmente se analiza cómo los ODS representan oportunidades para los emprendedores. Los hallazgos identifican el vínculo entre la oportunidad del emprendimiento como una solución al cambio climático y degradación ambiental, así como el papel que las universidades deberán desempeñar en este proceso.

Palabras clave: emprendimiento universitario; objetivos de desarrollo sostenible; sostenibilidad.

Abstract: University entrepreneurship emerges as a strategy for economic and social development, which sets a path to boost job creation, innovation and sustainable economic growth. The challenges that sustainability implies, embodied in the Sustainable Development Goals (SDG), symbolize areas of opportunity for entrepreneurs. The article identifies the challenges of university entrepreneurship against the SDGs. For this, first a review of the literature on entrepreneurship is made, entrepreneurship in Mexico is characterized and finally it is analyzed how the SDGs represent opportunities for entrepreneurs. The findings identify the link between entrepreneurship opportunity as a solution to climate change and environmental degradation, as well as the role that universities should play in this process.

Keywords: university entrepreneurship; sustainable development goals; sustainability.

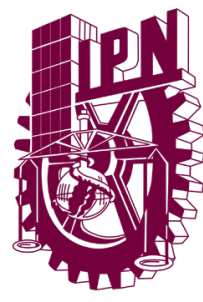

Pilar Pérez es Doctora en Economía y Gestión de la Innovación y Política Tecnológica de la Universidad Autónoma de Madrid e Investigadora del Centro de Investigaciones Económicas, Administrativas y Sociales (CIECAS) del Instituto Politécnico Nacional (IPN) de Méjico.

Contacto: mpperez@ipn.mx

Daniel Akenaton Granillo es Estudiante del Doctorado en Innovación en Ambientes Locales, Maestro en Política y Gestión del Cambio Tecnológico del Centro de Investigaciones Económicas, Administrativas y Sociales (CIECAS - IPN) y miembro del Registro CONACYT de Evaluadores Acreditados (RCEA).

Contacto: daakengh@gmail.com 


\section{Desafios do empreendedorismo universitário à frente dos Objetivos de Desenvolvimento Sustentável no México.}

Resumo: O empreendedorismo universitário surge como uma estratégia para o desenvolvimento econômico e social, que define um caminho para impulsionar a criação de empregos, a inovação e o crescimento econômico sustentável. Os desafios que a sustentabilidade implica, incorporados nos Objetivos de Desenvolvimento Sustentável (ODS), simbolizam áreas de oportunidade para os empreendedores. O artigo identifica os desafios do empreendedorismo universitário contra os ODS. Para isso, primeiro é feita uma revisão da literatura sobre empreendedorismo, o empreendedorismo no México é caracterizado e, finalmente, é analisado como os ODS representam oportunidades para os empreendedores. Os resultados identificam a ligação entre a oportunidade de empreendedorismo como uma solução para as mudanças climáticas e a degradação ambiental, bem como o papel que as universidades devem desempenhar neste processo.

Palavras-chave: empreendedorismo universitário; objetivos de desenvolvimento sustentável; sustentabilidade.

\section{Introducción}

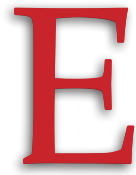

lemprendimiento es un proceso social en el que convergen campos del conocimiento que se complementan y retroalimentan. Su materialización en la creación de nuevos productos es solo la parte más visible de un sistema complejo donde intervienen diferentes actores, contextos, capacidades y recursos (Shane \& Venkataraman, 2000).

En Méjico, el bono demográfico se encuentra en la cúspide con casi 13 millones de habitantes, entre los 15 y los 29 años de edad. De acuerdo con el Instituto Nacional de Estadística y Geografía (INEGI, 2018a; 2018b), en 2015 había 22.3 millones de personas con estudios universitarios. En 2016, $43.6 \%$ de la población se encontraba en situación de pobreza (Coneval, 2018). Aunque, actualmente, los niveles de desempleo son bajos (3.4\% para el segundo trimestre de 2018), el sector informal representa el $56.9 \%$ de las personas ocupadas (OECD, 2018; INEGI, 2018a; 2018b). En ese contexto, la sociedad mejicana enfrenta el desafío de romper los círculos de la desigualdad, la pobreza y la informalidad, y brindar oportunidades laborales de calidad a esta generación.

La evidencia disponible sugiere que el emprendimiento es un elemento crucial para la generación de empleo, en particular, el asociado a empresas jóvenes. Sin embargo, este tipo de empresas son las más sensibles a los cambios y han desempeñado un rol importante en el crecimiento del empleo antes y después de las crisis financieras, tal como lo sugiere la 
entrada de nuevas empresas y las tasas de crecimiento más elevadas de las empresas jóvenes que sobreviven a episodios de recesión o crisis económica (OCDE, 2016).

La función social de los emprendedores requiere un contexto propicio para la creación de un círculo virtuoso de fomento de la actividad innovadora: mercados receptivos a nuevos productos, mercados laborales y de capital dinámicos y sistemas legales eficientes. Las empresas jóvenes, o startups, tienden a experimentar con tecnologías disruptivas y utilizar financiamiento externo para implementar y comercializar sus ideas, es por ello que pueden beneficiarse de las reformas de los mercados laborales y de capital al usar nuevas modalidades de financiamiento en etapa temprana, y bajo las condiciones adecuadas pueden contribuir al rejuvenecimiento del tejido productivo (Criscuolo, Gal \& Menon, 2014).

Existe un amplio consenso académico e institucional a nivel internacional sobre la estrecha vinculación entre bienestar económico, creación de empresas y actividad emprendedora. Las instituciones internacionales y los gobiernos buscan promover actividades para el nacimiento de startups (OCDE, 2016). Sin embargo, en Méjico la generación de empresas está fuertemente asociada al emprendimiento informal y la política pública aún contempla el emprendimiento universitario como un elemento secundario (Fairlie \& Woodruff, 2005).

El artículo analiza los desafíos del emprendimiento universitario en Méjico frente a los Objetivos de Desarrollo Sostenible (ODS). El documento se compone de tres secciones: en la primera se presenta el marco teórico del emprendimiento, seguido de una caracterización del emprendimiento en Méjico y finalmente se aborda el emprendimiento sostenible y las oportunidades para el desarrollo sostenible.

\section{Marco teórico del emprendimiento}

El emprendimiento emergió como un campo de investigación que ha cobrado mayor interés en las últimas tres décadas (Landstrom, Harirchi \& Astrom, 2012). Aún no hay un consenso sobre su significado. La propuesta de Shane \& Venkataraman (2000) presenta un análisis de cómo, quién y qué efectos y oportunidades son descubiertos, evaluados y explotados para crear nuevos bienes y servicios. Por su parte, Schumpeter (1947) se refiere al emprendimiento como un mecanismo de cambio económico de la sociedad, pues soluciona, vende, transgrede barreras rígidas del mercado, lidera y promueve su proyecto empresarial. 
En la literatura aparecen tres grandes acepciones sobre el concepto de emprendimiento. La primera lo considera como un potenciador de la creación de mercados mediante la difusión tecnológica, la innovación o la coordinación de mercados, por lo que se asume al emprendedor como un catalizador del crecimiento económico, de la creación de empleo y la competitividad (Etzkowitz \& Klofsten, 2005; Criscuolo, Gal \& Menon, 2014). La segunda aproximación se centra en la toma de riesgos como característica fundamental del emprendedor (Wennekers et al., 2005; York \& Venkataraman, 2010). El tercer enfoque pone de relieve la capacidad del emprendedor para introducir sus ideas en el mercado, enfrentando las incertidumbres y obstáculos inherentes, y la capacidad de gestionar sus propios negocios (OCDE, 2016). En cualquiera de las tres perspectivas antes mencionadas, se reconoce que los emprendedores son un motor endógeno de desarrollo de la sociedad.

La estructura productiva de un país y su productividad dependen de su capacidad para vincular el emprendimiento con objetivos clave de transformación productiva. El impulso del emprendimiento debe asumirse como una prioridad en el diseño de la política industrial y económica, debido a su potencial para mejorar las oportunidades de empleabilidad e ingresos entre grupos específicos, particularmente los jóvenes, y por ser un motor de la movilidad social (OCDE, 2016).

Si bien el emprendimiento es diverso, es posible clasificarlo en dos tipos principales: de subsistencia y de oportunidad. El primero alude a aquellos individuos que se involucran en actividades empresariales, pero que prefieren ser empleados asalariados antes que empresarios. En este tipo de emprendimiento se ubica un sector de trabajadores por cuenta propia y las pequeñas estructuras de negocios familiares. El emprendimiento de oportunidad, también llamado de alto impacto o transformacional, se refiere a aquellas empresas de alto crecimiento que cuentan con 10 empleados y un crecimiento medio de ventas o de empleados superior al $20 \%$ en sus primeros tres años de vida (Schoar, 2010).

El concepto de universidad emprendedora surge, en la década de los ochenta del siglo XX, del debate de las nuevas formas de generación de conocimiento y el papel de las Instituciones de Educación Superior (IES) ante los retos de la sociedad (Smilor y Sexton, 1987; Clark, 1998; Etzkowitz, 1983; Gibbons et al., 1994). De ese esfuerzo emana una conclusión clave: la universidad y su interacción con la sociedad requiere nuevas funciones (Etzkowitz, 2016; Wright, Birley \& Mosey, 2004; Klofsten et al., 2019). 
En 1980, la Ley Bayh-Dole de Estados Unidos respondió a las necesidades de una creciente demanda de investigación tecnológica por parte de los sectores más dinámicos y modernos (Tecnologías de Información y Comunicación, la nanotecnología y la biotecnología) que se encontraban en un estado incipiente (CEPAL, 2010). Algunos países como Alemania, Dinamarca y Japón introdujeron cambios al suprimir el "privilegio del profesor", que colocó a las IES como las titulares de los derechos de Propiedad Intelectual (PI) a cambio del derecho a una parte de las regalías para los inventores. Un número creciente de IES ha aceptado este desafío mediante la transformación de sus estructuras de incentivos, la participación en asociaciones de investigación y desarrollo (I+D), el establecimiento de empresas spin offy la comercialización de sus patentes (Wright et al., 2008).

Transferir la titularidad a las IES no fue suficiente para solucionar todos los problemas, la estrategia por sí sola no estimuló a los investigadores a convertirse en inventores (Cervantes, 2003). Hay evidencia que afirma que estos esfuerzos solo tuvieron efectos marginales, debido a que el proceso de TT requiere de un contexto propicio tanto al interior como fuera de las IES, por lo que fue necesario replantearse que el éxito de Estados Unidos no puede ser imitado con tan solo cambiar la legislación sobre PI, sino que el cambio debe ir acompañado de una estructura de incentivos y cambios organizacionales profundos. La comercialización de resultados de investigación requiere robustas relaciones externas con el ecosistema (Leydesdorff \& Meyer, 2010; Lockett \& Wright, 2005; Acs, Szerb \& Lloyd, 2017; Belitski, Aginskaja \& Marozau, 2019).

A pesar de las controversias, los datos muestran un crecimiento elevado en la comercialización de tecnología por las IES, tanto en países desarrollados como en los emergentes (Jaffe et al., 2007; WIPO, 2011). América Latina no ha experimentado una regulación como la Ley Bayh-Dole y el sistema "privilegio del profesor", tal como ocurrió en Estados Unidos y Alemania (Belitski, Aginskaja \& Marozau, 2019).

Actualmente, el rol de las universidades es multifacético y comienzan a implementar cambios de largo aliento para ser más emprendedoras y promover la comercialización de su conocimiento. La comprensión de los roles actuales de la universidad emprendedora es un prerrequisito para apreciarlas como la llave facilitadora en la TT, la innovación y el desarrollo económico, lo que propicia un cambio en los agentes que contribuyen a la competitividad de la sociedad (Klofsten et al., 2019; Belitski, Aginskaja \& Marozau, 2019). 
Las universidades líderes han expandido sus roles al ampliar las relaciones externas (Etzkowitz, 2016) y han encontrado nuevos caminos para promover su valor en la sociedad y su derecho a disponer de recursos. En países con escasa tradición emprendedora, las IES tienen un papel central en el fomento de la cultura emprendedora entre los estudiantes universitarios, con la intención de despertar en ellos una visión empresarial que les brinde herramientas para la toma de decisiones ante el deseo de abrir una empresa y mantenerla en el mercado (Hernández y Arano, 2015).

El análisis del emprendimiento académico se centra en el involucramiento de los académicos e investigadores en el desarrollo comercial de sus invenciones (Smilor \& Sexton, 1987; Mowery, 2005). Diversos estudios muestran una correlación significativa y positiva entre la productividad científica y los emprendimientos exitosos (Van Looy et al., 2011), así como entre el emprendimiento académico y el crecimiento económico. El modelo actual de emprendimiento universitario ha resaltado de estas relaciones, en particular, cuando el conocimiento creado en las IES deriva en alguna innovación con efectos en el crecimiento económico, lo cual viene aparejado de las funciones de docencia, investigación y difusión (Guerrero, Cunningham \& Urbano, 2015; Arocena \& Sutz, 2005).

\section{Características del emprendimiento universitario en Méjico}

El emprendimiento universitario en países en proceso de industrialización está fragmentado tanto en la práctica como en su estudio (Kantis, Ishida \& Komori, 2002). Resulta imprescindible generar datos y estrategias sinérgicas respecto a los mecanismos de TT desde las universidades, la regulación, los incentivos, la cultura e inversión en Investigación y Desarrollo Tecnológico e Innovación (I+D+I). Sin embargo, ha habido una proliferación de las oficinas de TT e incubadoras de empresas como figuras novedosas (Belitski, Aginskaja \& Marozau, 2019) y se ha ido construyendo un mayor entramado de mecanismos de apoyo a la TT. En el gráfico 1 se ilustra la evolución de la estructura de incentivos y mecanismos de apoyo para los emprendedores.

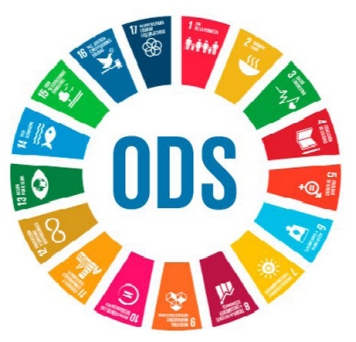


Gráfico 1. Evolución de los apoyos públicos para el emprendimiento universitario

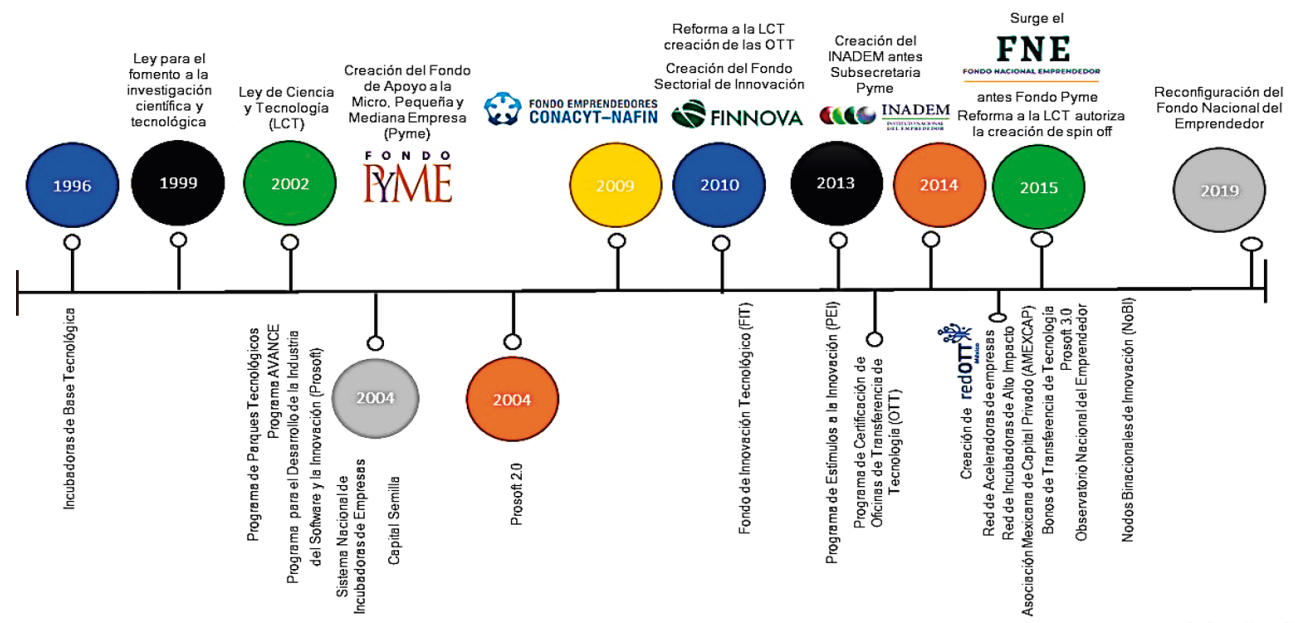

Fuente: Elaboración propia (2019).

En esta evolución se pueden identificar tres fases:

a) Durante la década de los noventa se dieron algunas iniciativas de vinculación entre la academia, la empresa y el surgimiento de Programa de Incubación de Empresas de Base Tecnológica, donde los esfuerzos eran aislados y liderados por las IES.

b) Con el cambio hacia una política empresarial, a partir de 2001, y la Ley de Ciencia y Tecnología, en 2002, se empezaron a diseñar políticas públicas encaminadas a la promoción de la TT y la innovación a partir de un enfoque sistémico, abierto a toda la sociedad, desde el emprendimiento social hasta el de alto impacto; así mismo, se generaron diferentes mecanismos como Aceleradoras de Negocios, programas de emprendedores, capital semilla, Incubadoras y aceleradoras de empresas, clústeres, parques tecnológicos y OTT, con la ayuda de programas como el FIT para empresas de base tecnológica y el Avance para proyectos de mayor contenido tecnológico.

c) En 2016 se da un cambio con la reforma en la Ley de Ciencia y Tecnología que autorizó la creación de empresas spin off emanadas de los resultados de investigación en la IES, se reconoció que los académicos requieren mecanismos de apoyo para la vinculación y transferencia de conocimiento. A partir de acá se creó el programa Nodos Binacionales de Innovación, que buscó acercar los tecnológicos a las demandas del mercado. 
Fue así como se desarrolló el entramado institucional que buscó fomentar la actividad innovadora y la TT en las IES. Estas modificaciones representan una oportunidad para que los investigadores participen de manera activa, difundan y comercialicen las innovaciones que surgen de sus investigaciones, de las que anteriormente no podían beneficiarse. Lo anterior pone a prueba las capacidades operativas e institucionales que cada IES ha desarrollado para TT y crear empresas spin off basadas en los resultados de la investigación que generan. En la tabla 1 se muestran algunos indicadores de la situación de l+D+l y del emprendiendo en Méjico.

Tabla 1. Numeralia del I+D+l y emprendimiento en Méjico

\begin{tabular}{|l|r|}
\hline \multicolumn{1}{|c}{ Variable } & \multicolumn{1}{c|}{ Registro } \\
\hline Población (2015). & $119,938,473$ \\
\hline Población (18-64 años en porcentaje). & $65.20 \%$ \\
\hline Acervo de Recursos Humanos en Ciencia y Tecnología (RHCyTE) en 2017. & $11,849,581$ \\
\hline $\begin{array}{l}\text { Personal ocupado en alguna actividad científica o tecnológica } \\
\text { (\% de RHCyTE) 2017. }\end{array}$ & 70.5 \\
\hline Personal dedicado a otras funciones (\% de RHCyTE). & 29.5 \\
\hline Egresados de licenciatura 2017. & 631,454 \\
\hline Alumnos graduados de maestría en 2017. & 87,772 \\
\hline Alumnos graduados de doctorado en 2017. & 9,628 \\
\hline Promedio de edad para emprender (años). & $25-34$ \\
\hline Emprendedores jóvenes (16-29 años) de subsistencia (\%) en 2016. & 37.3 \\
\hline Emprendedores jóvenes (16-29 años) de necesidad (\%) en 2016. & 61.7 \\
\hline Emprendimiento femenino (Mujeres empleadoras) (\%) en 2006. & 20.8 \\
\hline $\begin{array}{l}\text { Gasto en Investigación y Desarrollo Experimental (GIDE) respecto } \\
\text { del Producto Interno Bruto en Méjico, 2017 (\%). }\end{array}$ & 0.48 \\
\hline Número de patentes nacionales concedidas en Méjico (2018). & 9,634 \\
\hline Número de solicitud de patentes universitarias (acumulado 2013-2018). & 1,976 \\
\hline Número de empresas internas incubadas universitarias (ITESM) 2010. & 585 \\
\hline Número de empresas internas incubadas universitarias (IPN) 2010. & 40 \\
\hline Número de empresas externas incubadas universitarias (ITESM) 2010. & 539 \\
\hline Número de empresas externas incubadas universitarias (IPN) 2010. & 85 \\
\hline
\end{tabular}

Fuente: INEGI (2019), CONACYT (2016; 2018), INCAE (2016), RedEmprendia (2014).

Los éxitos y aprendizajes de otras regiones influyeron en la creación de condiciones para el surgimiento de diferentes iniciativas que fomentaban el emprendimiento y, en menor medida, el emprendimiento universitario. 
Por su parte, investigadores del Global Entrepreneurship Monitor (GEM) reportaban que la actividad de emprendimiento en países en desarrollo era mayor que en los desarrollados (Vivarelli, 2013), en el caso mejicano, los estudios sobre emprendimiento universitario son fragmentados (Fairlie \& Woodruff; 2005; Cantú-Ortiz et al., 2017; Guerrero et al., 2016; Pérez y Calderón, 2019).

La evidencia en Méjico muestra que la difusión, capacitación y apoyos para fomentar el emprendimiento y afianzar las empresas creadas son escasos. Para que las empresas innovadoras permanezcan en su actividad falta más difusión de los apoyos a los que se puede acceder. Aunque la percepción del emprendedor presenta avances considerables, es necesario promover una cultura emprendedora que difunda los aportes y beneficios para los individuos y para la solución de los grandes problemas nacionales.

Otros elementos estructurales como la piratería, la corrupción y la regulación excesiva representan obstáculos para las Pyme (Observatorio Nacional del Emprendedor, 2015). La falta de información acerca de oportunidades de financiamiento, la baja inversión en investigación y desarrollo para impulsar la innovación, la brecha entre las capacidades de la fuerza laboral y las necesidades del mercado, entre otros, desincentivan la creación de nuevos negocios y limitan su posibilidad de crecimiento. En general, el segmento que se ha atendido en Méjico es el emprendimiento social y juvenil con un perfil de subsistencia y autoempleo, aunque con rasgos y características distintas.

En esta ecuación, que apenas empieza a vislumbrarse en las políticas públicas del emprendimiento, hay un segmento que aún no se ha analizado: el grupo etario de 50 años y más. Muchos de los profesores universitarios en condiciones de emprender se encuentran en este rango de edad, además, representaría una opción para los jubilados, quienes tienen una esperanza de vida creciente debido al mejoramiento de las condiciones de vida, lo cual repercute en los sistemas de pensiones y servicios de salud. Los emprendedores mayores de 50 años tienen alta cualificación, mayor propensión al riesgo, innovación, iniciativa, autoeficacia y actitud ante a la autonomía, tal como sus contrapartes más jóvenes. A medida que la pirámide poblacional vaya envejeciendo, su incorporación como actores de cambio en la sociedad será mayor.

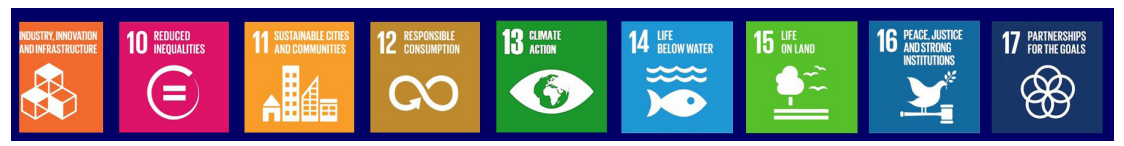




\section{Emprendimiento sostenible y las oportunidades para el desarrollo sostenible}

El rol del emprendimiento, como un vehículo para la trasformación económica y social, no es nuevo en la literatura (Drucker, 1985; Schumpeter 1942). En este sentido, ha figurado como un canal importante para la generación de productos y servicios sostenible y el surgimiento de nuevos proyectos, incluso se ha asumido como una panacea para muchos de los problemas sociales y ambientales. Los Objetivos de Desarrollo Sostenible (ODS), que emergieron de la Conferencia de Rio+20, apuntan al mejoramiento de las condiciones económicas, sociales y ambientes de los países menos desarrollados; sin embargo, no hay estudios que exploren las posibilidades del emprendimiento para lograr esas metas. La conciencia de la sostenibilidad que Naciones Unidas ha impulsado desde la Conferencia de Rio+20, en 2012, y la creciente necesidad de un cambio en la producción de energía y en el uso de los recursos naturales, ha propiciado una mayor atención en el cambio climático y en la degradación del medio ambiente (Dhahri \& Omri, 2018).

Retomando a la World Commission on Environment and Development (WCED, 1987) se considera desarrollo sostenible "como un desarrollo en el cual las necesidades de la generación actual satisfacen sus necesidades sin comprometer la capacidad de las generaciones futuras" (p.43). El desarrollo sostenible es reconocido como un camino para reorientar el desarrollo hacia un modelo más inclusivo, en el que se busca una relación simbólica entre los sistemas económicos, sociales y ambientales para las generaciones presentes y futuras (Dhahri \& Omri, 2018).

Un número creciente de universidades ha respondido a la obligación ética de integrar sistemáticamente la sostenibilidad dentro de sus instituciones a través de la enseñanza, investigación, operación, evaluación e informes (Lozano et al., 2013). Este fenómeno surge recientemente como tema de investigación para identificar aquellas universidades que son sostenibles (Disterheft et al., 2015; Figuero \& Raufflet, 2015; Ramos et al., 2015; Fichter \& Tiemann, 2018).

Pese a que el emprendimiento resulta prometedor frente a la sostenibilidad y reducción del cambio climático, al ofrecer productos y servicios sostenibles y la implementación de nuevos proyectos relacionados con las preocupaciones ambientales y sociales, su rol y naturaleza son inciertos. Por otra parte, el tema de la sostenibilidad en la literatura del emprendimiento es escaso (Hall et al., 2010; Dhahri \& Omri, 2018). 
Aunque el emprendimiento es reconocido recientemente como un vehículo que permite una economía más sostenible, la importancia de los emprendedores como vehículos del cambio social y económico no es nuevo en la literatura; sin embargo, existen lagunas en el conocimiento (O'Neil et al., 2009). Así mismo, se busca explicar cómo la catálisis de la relación entre instituciones, emprendimiento y sostenibilidad en los países puede generar productos y servicios más sostenibles (Silvestre, 2015) y plantear la necesidad de una mayor demanda por parte de la sociedad para este tipo de iniciativas, tanto en términos de educación, empresas y políticas públicas (Almeida, et al., 2013; Lozano et al., 2013). El reconocimiento del emprendimiento y la innovación como una solución puede ayudar a reducir la desigualdad y la contaminación (York \&Venkataraman, 2010), además, enfatiza la importancia de las economías sostenibles (Ben Youssef, Boubaker \& Omri, 2018).

Resulta necesario promover la oportunidad del emprendimiento como una solución plausible de la degradación ambiental y del cambio climático. Los emprendedores son conscientes de la existencia de un importante potencial de mercado para productos "amigables con el ambiente", que han tenido un auge creciente tanto países en vías de desarrollo como en los desarrollados. Una nueva generación de emprendedores, apoyados por las nuevas tecnologías, comienza a aprovechar ese nuevo nicho de oportunidades. En algunos casos, los emprendedores se enfrentan a una poderosa regulación, lo que propicia el uso de métodos de producción más sostenibles y les permite incrementar su participación en el mercado y entrar a nuevos mercados (Ben Youssef, Boubaker \& Omri, 2018).

La necesidad de incluir el tema de la sostenibilidad responsable en la educación de los líderes ha sido enfatizada en la literatura (Lozano et al., 2006). Estudios recientes sugieren que un creciente número de universidades consideran que la sostenibilidad debe estar integrada al sistema de soporte del emprendimiento, o que la sostenibilidad debe concretarse en actividades implementadas y promovidas con espíritu emprendedor (Fichter \& Clausen, 2016). Estos hallazgos hablan positivamente de la demanda de los policy makers de un emprendimiento sostenible como parte de la educación de los "emprendedores verdes" del futuro, quienes deberán adoptar esta mentalidad para ayudar a identificar oportunidades de negocios sostenibles, mediante nuevas formas de cooperación entre la academia y la industria (European Commission, 2014; Fichter \& Tiemann, 2018). 
Gráfico 2. Papel de la Universidad emprendedora sostenible

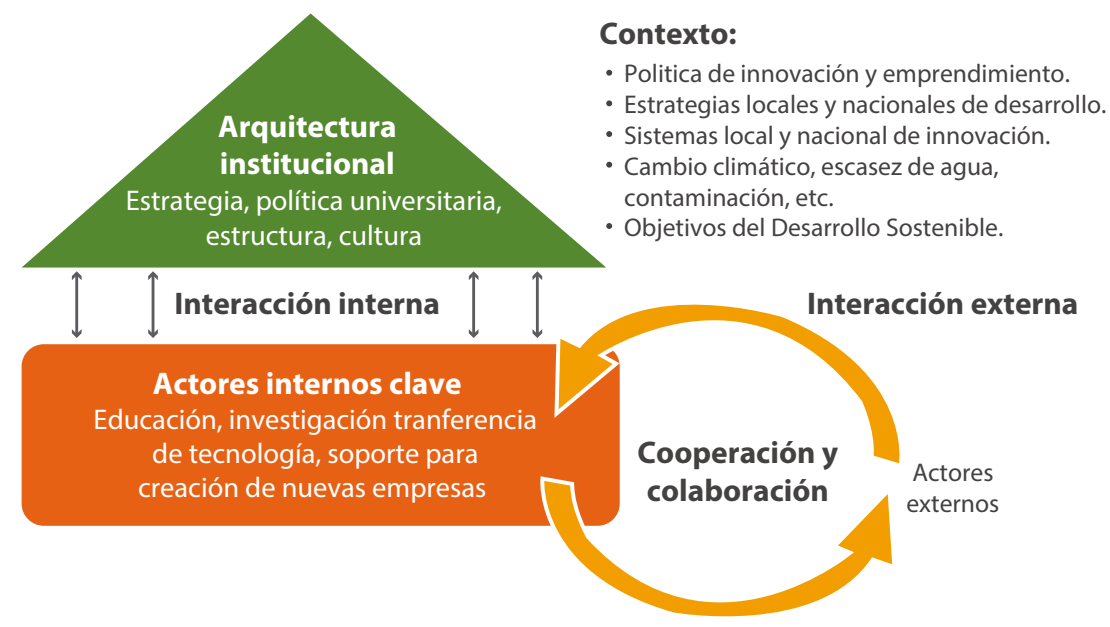

Fuente: Elaboración propia con base en Fichter \& Tiemann (2018).

Los ODS contienen los principales retos que enfrenta la sociedad actual, tales como la reducción de la pobreza, el combate al cambio climático, la gestión del agua, la salud, la educación, la equidad, entre otros. Los ODS son importantes porque plasman las necesidades sociales y buscan promover el desarrollo internacional (Galli et al., 2018).

En la construcción de los ODS fueron consultados líderes mundiales, expertos en diversos temas, representantes de Organizaciones No Gubernamentales (ONG) y representantes del sector privado. Las razones que motivan la participación empresarial derivan de la crisis económica de 2008 y su consecuente modificación en los presupuestos de los gobiernos y los retos globales (Scheyvens et al., 2016). Otras de las causas es el cambio de los patrones de consumo hacia la demanda de productos y servicios sostenibles, lo que ha propiciado una mayor presión en las empresas para desarrollar innovaciones sostenibles (Von Geibler et al., 2019). Aunado a esto, existe una fuerte expectativa en la capacidad de las empresas para generar innovaciones y nuevas tecnologías que permitan resolver problemas relacionados con los ODS (Ritala, 2019).

Sin embargo, las críticas hacia el sector privado y su influencia y capacidad en resolver los problemas planteados por los ODS (Ritala, 2019; Scheyvens, et al., 2016) están latentes. De acuerdo con Ritala (2019), hay diferentes posicionamientos al respecto, que van de un optimismo exacerbado que considera a las empresas como los agentes que pueden conducir un cambio respecto a los ODS, a la anulación de sus posibilidades para plantear 
soluciones. Entre los señalamientos hacia las empresas se destacan: 1) la agenda neoliberal en los mercados y la estructuración de las políticas públicas, 2) el distanciamiento para atender problemas estructurales, 3) los problemas para trabajar en "equipo" con otras empresas, 4) los modelos de negocio empresariales con una visión de corto plazo, 5) la preferencia de las empresas por los rendimientos frente las necesidades sociales y 6 ) diferencias relacionadas con la sustentabilidad entre la academia y las empresas (Ritala, 2019; Scheyvens et al., 2016).

Las investigaciones de los ODS y la innovación están relacionadas con las innovaciones sociales, las cuales han ido generando un mayor interés de la academia en los últimos años, con el fin de identificar cuáles son y cuál es su relación con el emprendimiento y el sector privado. Así mismo, se han ubicado como actores que guían dichas innovaciones sociales a los emprendedores sociales, ONG, instituciones públicas, empresas y grupos de ciudadanos preocupados por los diferentes problemas que enfrentan, pero que no necesariamente se alinean a los ODS (Eichler \& Schwarz, 2019).

Sin embargo, no solo las empresas enfrentan problemas para atender los ODS, los sistemas educativos en sus niveles básicos deben incluir una mayor concientización sobre la importancia de los ODS y sus efectos dentro de la sociedad, así como incidir en la formación de emprendedores con una fuerte inclinación a resolver algún problema relativo con los ODS (Chirambo, 2018). Lo anterior obliga a los gobiernos a incorporar la promoción de la cultura emprendedora en los sistemas educativos y de transmisión del conocimiento, en un contexto que resalte la capacidad de resolver alguna necesidad social. Así mismo, se enfatiza la exigencia de innovar la forma en que se transmite y genera conocimiento, no solo en las estructuras públicas sino en la educación privada.

En cuanto a los emprendedores, se les debe dotar de elementos que les permitan generar propuestas de mercado para resolver o atender alguno de los retos de los ODS. Para Von Geibler et al. (2019) los ODS pueden generar barreras cuando se intenta innovar en estas áreas, es por esto que proponen una herramienta de gestión tecnológica para la valiadación de las nuevas propuestas en etapas tempranas de la madurez de las innovaciones, relacionadas con los Technology Readness Levels (TRL). Esta herramienta permite a los emprendedores obtener información en etapas previas a la realización de una propuesta de producto o servicio, y coadyuva a disminuir el tiempo de la generación y a obtener información útil para su realización.

Han sido documentadas diferentes propuestas y experiencias relacionadas con la solución de los ODS, donde el desarrollo de innovaciones ha 
cobrado importancia y presenta una participación social activa de los emprendedores a través de sus propuestas integrales. Estas propuestas están relacionadas con la creación de cadenas de valor en la agricultura (Biénabe, et al., 2017), el uso sostenible de los recursos y medioambiente (Lehmann, 2018; Zielinski et al., 2018) y uso y manejo del agua (Lagerstedt et al., 2017; Gebauer \& Jennings Saul, 2014; Bakker, 2014). En Méjico se han identificado una serie de startups enfocadas en la atención de los ODS, las propuestas abarcan la atención de la salud, energias limpias, democratización de la educación, entre otros (tabla 2), y buscan atender problemáticas sociales que no han sido consideradas y que pueden generar beneficios económicos, sociales y de empleo.

En el caso de Méjico, los esfuerzos de las IES por incorporar los tema de sostenibilidad han ido creciendo, desde programas de sensibilicacion y reeducación, hasta carreras completas y posgrados (tanto univesidades públicas como privadas, nacionales y regionales). Como resultado de la confluencia tanto de la cultura emprendedora y la incorporación de la sosteniblidad como parte de las áreas de oportunidad se han identificado una serie de empresas startusp que fueron creadas por universitarios y que tienen una relación directa con los ODS.

Tabla 2. Emprendimientos disruptivos en Méjico asoicados a los ODS

\begin{tabular}{|l|l|l|}
\hline \multirow{2}{*}{ Emprendimiento } & \multicolumn{1}{|c}{ Necesidad que resuelve } & \multicolumn{1}{c|}{$\begin{array}{c}\text { Solución } \\
\text { propuesta }\end{array}$} \\
\hline Blooders & $\begin{array}{l}\text { Plataforma para conectar donadores } \\
\text { de sangre con quienes la necesitan, } \\
\text { haciendo el proceso más eficiente. }\end{array}$ & $\begin{array}{l}\text { Donación altruista } \\
\text { de sangre. }\end{array}$ \\
\hline Isla urbana & $\begin{array}{l}\text { Diseño e implementación de sistemas } \\
\text { de tratamiento y aprovechamiento } \\
\text { de agua con biotecnología. }\end{array}$ & $\begin{array}{l}\text { Saneamiento } \\
\text { y el acceso libre } \\
\text { al agua. }\end{array}$ \\
\hline Delta Signals & $\begin{array}{l}\text { Desarrollo de productos relacionados } \\
\text { con el uso eficiente del agua } \\
\text { proveniente de captación de lluvia. }\end{array}$ & $\begin{array}{l}\text { Uso de agua de } \\
\text { captación de lluvia. }\end{array}$ \\
\hline INDI & $\begin{array}{l}\text { Tecnología para prevención de } \\
\text { desastres identificando áreas de riesgo. }\end{array}$ & $\begin{array}{l}\text { Prevención } \\
\text { de desastres. }\end{array}$ \\
\hline Robin & $\begin{array}{l}\text { Diseño interactivo de productos } \\
\text { relacionados con la salud. }\end{array}$ & $\begin{array}{l}\text { Mejora } \\
\text { en la atención } \\
\text { de la salud. }\end{array}$ \\
\hline Cronology & $\begin{array}{l}\text { Aplicación que enseña a niños } \\
\text { educación financiera, simulación } \\
\text { de ahorro. Alcancía digital, etc. }\end{array}$ & $\begin{array}{l}\text { Educación financiera } \\
\text { de los niños. }\end{array}$ \\
\hline & $\begin{array}{l}\text { Fabricación de papel a base de residuos } \\
\text { de PET. }\end{array}$ & Reciclaje de PET. \\
\hline
\end{tabular}




\begin{tabular}{|l|l|l|}
\hline \multicolumn{2}{|c|}{ Emprendimiento } & \multicolumn{2}{c|}{$\begin{array}{c}\text { Solución } \\
\text { propuesta }\end{array}$} \\
\hline Quotanda & $\begin{array}{l}\text { Plataforma financiera estudiantil cuya } \\
\text { misión es democratizar el acceso a la } \\
\text { educación a través de la mejora en las } \\
\text { opciones de financiación estudiantil. }\end{array}$ & $\begin{array}{l}\text { Democratización } \\
\text { del acceso } \\
\text { a la educación. }\end{array}$ \\
\hline LuminaKret & $\begin{array}{l}\text { Producción de materiales } \\
\text { de construcción sostenibles. }\end{array}$ & $\begin{array}{l}\text { Reducción } \\
\text { de la contaminación } \\
\text { ambiental } \\
\text { yalta resistencia } \\
\text { estructuras } \\
\text { de concreto. }\end{array}$ \\
\hline Xintiba & $\begin{array}{l}\text { Desarrollo de videojuegos terapéuticos, } \\
\text { especializados en niños con autismo. }\end{array}$ & $\begin{array}{l}\text { Mejora en la } \\
\text { educación de niños } \\
\text { con autismo. }\end{array}$ \\
\hline Strap Technologies & $\begin{array}{l}\text { Desarrollo de dispositivos para detectar } \\
\text { obstáculos y generar avisos a personas } \\
\text { con discapacidad visual. }\end{array}$ & $\begin{array}{l}\text { Independencia de } \\
\text { las personas con } \\
\text { discapacidad visual. }\end{array}$ \\
\hline DerTek & $\begin{array}{l}\text { Producción y comercialización de } \\
\text { biocombustibles avanzados en } \\
\text { comunidad de alta vulnerabilidad. }\end{array}$ & $\begin{array}{l}\text { Reducción } \\
\text { de la contaminación } \\
\text { ambiental } \\
\text { provocada } \\
\text { por el uso } \\
\text { de combustibles } \\
\text { fósiles en vehículos } \\
\text { automotores. }\end{array}$ \\
\hline
\end{tabular}

Fuente: Elaboración propia basado en Entrepreneur (2016), BID (2017), Forbes (2013) y Disruptivo tv (2015).

\section{Conclusiones}

La innovación es fundamental para los individuos, las organizaciones, la sociedady parala construcción de unfuturosostenible.Lasobreexplotación de los recursos del planeta no permitirá que se mantenga el ritmo de crecimiento en el mediano y largo plazo. La creciente sobrepoblación y la desigualdad están exigiendo que los alimentos, los servicios y otro tipo de bienes se produzcan de forma más eficiente. Se requieren cambios importantes para que el emprendimiento figure como una estrategia trasversal del desarrollo sostenible, independientemente del sector etario para valorizar el talento de los ciudadanos.

La única forma de frenar la destrucción del planeta es producir mejor con menos recursos y considerar la desigualdad. La innovación frugal permite innovar a través del ingenio. Cuando existen recursos limitados los emprendedores explotan al máximo su creatividad. Es importante introducir este tipo de filosofía de innovación, donde las IES desempeñan un papel fundamental como centros de capacitación y formación de 
emprendedores, y como generadoras de empresas de alto impacto social. Las IES tienen que asumir la tarea de generar las soluciones que la sociedad requiere. Así mismo, los ODS representan oportunidades para innovar, tanto en los productos y servicios como en los modelos de negocio, donde debe considerarse el papel activo de las empresas como agentes de cambio frente a los problemas que se plantean.

Los gobiernos, el de Méjico en particular, deben modificar sus políticas públicas, no solo para hacer frente a los retos identificados por los ODS, sino para realizar cambios profundos relacionados con la cultura emprendedora, el desarrollo de negocios sostenibles, la sensibilización frente al cambio climático, el impulso a emprendedores con modelos de negocios de impacto social y la modificación en la estructura educativa, con el fin de generar y transmitir conocimientos que faciliten la atención de estos objetivos.

En el actual contexto de cambio de gobierno en Méjico hay incertidumbre respecto a las políticas públicas de apoyo a la creación de empresas y valorización del conocimiento generado en las IES. Sin embargo, lejos de la retórica que puede envolver estos temas, deber quedar claro que el emprendiendo y la innovación son imprescindibles para el desarrollo económico y social del país.

\section{Referencias bibliográficas}

1. ACS, Zoltan, SZERB, László \& LLOYD, Ainsley. (2017). Mapping the global entrepreneurial ecosystem. Global Entrepreneurship and Development Index 2017. $1^{\text {a }}$ ed. Washington: Springer. 119 pp.

2. AROCENA, Rodrigo \& SUTZ, Judith. (2005). Latin American Universities: From an Original Revolution to an Uncertain Transition. Higher Education, Vol. 50. Pp. 573-592. https://doi.org/10.1007/s10734-004-6367-8

3. ALMEIDA, Cecilia, BONILLA, Silvia, GIANNETTI, Biagio \& HUISINGH, Donald. (2013). Cleaner Production initiatives and challenges for a sustainable world: an introduction to this special volume. Journal Clean Production, Vol. 47, №. 1. Pp. 1-10. https://doi. org/10.1016/j.jclepro.2013.03.010

4. BAKKER, Karen. (2014). The Business of Water: Market Environmentalism in the Water Sector. Annual Review of Environment and Resources. Vol. 39. №. 1. Pp. 469-494. https://doi.org/10.1146/annurev-environ-070312-132730

5. BELITSKI, Maksim, AGINSKAJA, Anna \& MAROZAU, Radzivon. (2019). Commercializing university in transition economies: technology transfer offices or direct industrial funding. Research Policy, Vol. 48, №. 3. Pp. 601-615. https://doi.org/10.1016/j. respol.2018.10.011 
6. BEN, Youssef, BOUBAKER, Adel \& OMRI, Anis. (2018). Entrepreneurship and sustainability: the need for innovative and institutional solutions. Technological Forecasting \& Social Change, Vol. 129, №. 1. Pp. 232-241. https://doi.org/10.1016/j. techfore.2017.11.003

7. BID (2017). 13 startups Disruptivas de América Latina que usan la creatividad para mejorar sus vidas. Disponible en Internet: https://blogs.iadb.org/industrias-creativas/ es/13-startups-disruptivas-de-america-latina-y-el-caribe-que-estan-usando-lacreatividad-para-mejorar-vidas/ Consultado: 04.12.2019.

8. BIÉNABE, Estelle, RIVAL, Alain \& LOEILLET, Denis. (2017). Sustainable Development and Tropical Agri-chains. $1^{\text {a }}$ ed. Dordrecht: Springer. 357 pp.

9. CANTÚ-ORTIZ, Francisco Javier, GALEANO, Nathalíe, MORA-CASTRO, Patricia \& FANGMEYER, James. (2017). Spreading academic entrepreneurship: Made in Mexico. Business Horizons, Vol. 60, №. 4. Pp. 541-550. https://doi.org/10.1016/j. bushor.2017.04.002

10. CERVANTES, Mario. (2003). Universidades y organismos públicos de investigación: utilización de la propiedad intelectual, concretamente las patentes, para promover la investigación y crear start-ups innovadoras. Disponible en Internet: http://www.wipo. int/sme/es/documents/academic_patenting.htm Consultado: 03.12.2019.

11. CHIRAMBO, Dumisani. (2018). Leaving No-One Behind: Improving Climate Change and Entrepreneurship Education in Sub-Saharan Africa Through E-Learning and Innovative Governance Systems (pp. 33-47). En: AZEITEIRO, Ulises; LEAL FILHO, Walter y AIRES, Luisa (Edits.), Climate Literacy and Innovations in Climate Change Education. Suiza: Springer International Publishing.

12. CLARK, Mario Burton. (1998). Creating Entrepreneurial Universities: Organizational Pathways of Transformation. $1^{\text {a }}$ ed. Nueva York: Pergamon Press. 180 pp.

13. CEPAL. (2010). Espacios Iberoamericanos. Vínculos entre universidades y empresas para el desarrollo tecnológico. $1^{\mathrm{a}} \mathrm{ed}$. Santiago de Chile: CEPAL-CEGIB. 108 pp.

14. CRISCUOLO, Chiara, GAL, Peter \& MENON, Carlo. (2014). The dynamics of employment growth: New evidence from 18 countries. OECD Science, Technology and Industry Policy Papers, Vol. 14. №. 1. Pp. 1-96. https://doi.org/10.1787/5jz417hj6hg6-en

15. DHAHRI, Sabriney OMRI, Anis. (2018). Entrepreneurship contribution to the three pillars of sustainable development: What does the evidence really say? World Development, Vol. 106, №. 1. Pp 64-77. https://doi.org/10.1016/j.worlddev.2018.01.008

16. DISRUPTIVOTV. (2015). Startup México presenta sus 33 nuevas Startups. Disponible en Internet: https://disruptivo.tv/columnas-y-notas/graduacion-startup/ Consultado: 02.12.2019.

17. DISTERHEFT, Antje, CAEIRO, Sandra, AZEITEIRO Ulisses \& FILHO, Walter. (2015). Sustainable universities. A study of critical success factors for participatory approaches. Journal Clean Production, Vol. 106, №. 1. Pp. 11-21. https://doi.org/10.1016/j. jclepro.2014.01.030

18. DRUCKER, Peter Ferdinand. (1985). La innovación y el empresario innovador: la práctica y los principios. $1^{\text {a }}$ ed. Barcelona: Edhasa, 307 pp.

19. EICHLER, Georg \& SCHWARZ, Erich. (2019). What sustainable development goals do social innovation address? A systematic review and content analysis of social innovation literature. Sustainability, Vol. 11, №. 522. Pp. 3-18. https://doi.org/10.3390/su11020522 
20. ENTREPRENEUR (2016). Estas son las 14 startups más disruptivas en América Latina, Disponible en Internet: https://www.entrepreneur.com/article/282420 Consultado 02.12.2019.

21. ETZKOWITZ, Henry. (2016). The entrepreneurial university: vision and metrics. Industry and Higher Education, Vol. 30, №. 2. Pp. 83-97. https://doi.org/10.5367/ihe.2016.0303

22. ETZKOWITZ, Henry. (1983) Entrepreneurial scientist and entrepreneurial universities in American academic science. Minerva, Vol. 21, №. 2-3. Pp. 198-233. Disponible en Internet: https://www.jstor.org/stable/41820527?seq=1

23. ETZKOWITZ, Henry \& KLOFSTEN, Magnus. (2005). The innovating region: toward a theory of knowledge-based regional development. R\&D Management, Vol. 35, №. 3. Pp. 246-255. https://doi.org/10.1111/j.1467-9310.2005.00387.x

24. EUROPEAN COMMISSION (2014). Green Action Plan for SMEs: Enabling SMEs to turn environmental challenges into business opportunities. Disponible en Internet: https:// ec.europa.eu/growth/content/green-action-plan-smes-turning-environmentalchallenges-business-opportunities-0_en Consultado: 29.11.2019.

25. FAIRLIE, Robert \& WOODRUFF, Christopher. (2005). Mexican Entrepreneurship: A Comparison of Self-Employment in Mexico and the United States. Working paper 11527 (pp. 123-158). En:BORJAS, George J. Mexican Immigration to the United States. Cambridge: University of Chicago Press - National Bureau of Economic Research.

26. FICHTER, Klaus \& TIEMANN, Irina. (2018). Factors influencing university support for sustainable entrepreneurship: Insights from explorative case studies. Journal Clean Production, Vol. 175 №. 1. Pp. 512-524. https://doi.org/10.1016/j.jclepro.2017.12.031

27. FICHTER, Klaus \& CLAUSEN, Jens. (2016). Diffusion dynamics of sustainable innovationinsights of diffusion patterns based on the analysis of 100 sustainable product and service innovation. Journal Innovation Management, Vol. 4, №. 2. Pp. 30-67. https:// doi.org/10.24840/2183-0606_004.002_0004

28. FIGUEIRÓ, Paola \& RAUFFLET, Emmanuel. (2015). Sustainability in high education: systematic review with focus on management education. Journal Clean Production, Vol. 106, №. 1. Pp. 22-33. https://doi.org/10.1016/j.jclepro.2015.04.118

29. FORBES (2013). 10 startup mexicanas listas para cambiar al mundo, Disponible en Internet: forbes.com.mx/10-startups-mexicanas-listas-para-cambiar-el-mundo/ Consultado: 28.11.2019.

30. GALLI, Alessandro, ĐUROVIĆ, Gordana, HANSCOM, Laurel \& KNEŽEVIĆ, Jelena. (2018). Think globally, act locally: Implementing the sustainable development goals in Montenegro. Environmental Science and Policy, Vol. 84, №.1. Pp. 159-169. https://doi. org/10.1016/j.envsci.2018.03.012

31. GEBAUER, Heiko \& JENNINGS SAUL, Caroline. (2014). Business model innovation in the water sector in developing countries. Science of the Total Environment, Vol. 488-489, №. 1. Pp. 512-520. https://doi.org/10.1016/j.scitotenv.2014.02.046

32. GIBBONS, Michael, LIMOGES, Camille, NOWOTNY, Helga, SCHWARTZMAN, Simon, SCOTT, Peter \& TROW, Martin. (1994). The New Production of Knowledge. $1^{\text {a }}$ ed. Londres: Sage Publications Ltd. 179 pp.

33. GUERRERO, Maribel, URBANO, David, FAYOLLE, Alain, KLOFSTEN, Magnus \& MIAN, Sarfraz. (2016). Entrepreneurial Universities: Emerging Models in the New Social and 
Economic Landscape. Small Business Economics, Vol. 47, №. 3. Pp. 551-563. https:// doi.org/10.1007/s11187-016-9755-4

34. GUERRERO, Maribel, CUNNINGHAM, James \& URBANO, David. (2015). Economic Impact of Entrepreneurial Universities' Activities: An Exploratory Study of the United Kingdom. Research Policy, Vol. 44, №.3. Pp. 748-764. https://doi.org/10.1016/j. respol.2014.10.008

35. HALL, Jeremy, DANEKE, Gregory \& LENOX, Michael. (2010). Sustainable development and entrepreneurship: past contributions and future directions. Journal of Business Venturing, Vol. 25, №. 5. Pp. 439-448. https://doi.org/10.1016/j.jbusvent.2010.01.002

36. HERNÁNDEZ, Carlos y ARANO, Raúl Manuel. (2015). El desarrollo de la cultura emprendedora en estudiantes universitarios para el fortalecimiento de la visión empresarial. Ciencia Administrativa, Vol. 1, №. 1. Pp. 28-37. Disponible en Internet: https://www.uv.mx/iiesca/files/2012/10/04CA201501.pdf

37. JAFFE, Adam B., LERNER, Josh, STERN, Scott \& THURSBY, Marie C. (2007). Academic Science and Entrepreneurship: Dual Engines of Growth? Journal of Economic Behavior \& Organization, Vol. 63 №. 4. Pp. 573-576. Disponible en Internet: https://www.nber. org/books/jaff05-2

38. KANTIS, Hugo, ISHIDA, Masahiko \& KOMORI, Mashahiko. (2002). Empresarialidad en economías emergentes: creación y desarrollo de nuevas empresas en América Latina y el Este de Asia. $1^{\text {a }}$ ed. Washington, D. C.: Banco Interamericano de Desarrollo. 134 pp.

39. KLOFSTEN, Magnus, FAYOLLE, Alain, GUERRERO, Maribel, MIAN, Sarfraz, URBANO, David \& WRIGHT, Mike. (2019). The entrepreneurial university as driver for economic growth and social change- Key strategic challenges. Technological forecasting \& social change. Vol. 141, №. 1. Pp. 149-159. https://doi.org/10.1016/j.techfore.2018.12.004

40. LAGERSTEDT, Jessica, AHLGREN, Kajsa \& BENGTSSON, Lars A. (2017). Joint business model innovation for sustainable transformation of industries e A large multinational utility in alliance with a small solar energy company. Journal of Cleaner Production, Vol. 160, №. 1. Pp.139-150. https://doi.org/10.1016/j.jclepro.2017.03.151

41. LANDSTROM, Hans, HARIRCHI, Gouya \& ASTROM, Fredick. (2012). Entrepreneurship: exploring the knowledge base. Research Policy, Vol .4, № 7 . Pp. 1154-1181. https://doi. org/10.1016/j.respol.2012.03.009

42. LEHMANN, Harry. (2018). Factor X. Challenges, Implementation Strategies and Examples for a Sustainable Use of Natural Resources. 1 ed. Suiza: Springer International Publishing AG. 452 pp.

43. LEYDESDORFF, Loet \& MEYER, Martin. (2010). The decline of university patenting and the end of the Bayh-Dole effect. Scientometrics, Vol. 83, №. 3. Pp. 355-362. https://doi. org/10.1007/s11192-009-0001-6

44. LOCKETT, Andy Geoffrey \& WRIGHT, Mike. (2005). Resources, capabilities, risk capital and the creation of university spin-out companies. Research Policy, Vol. 34, № 7. Pp. 1043-1057. https://doi.org/10.1016/j.respol.2005.05.006

45. LOZANO, Rodrigo, LUKMAN, Rebeca K., LOZANO, Francisco José, HUISINGH, Donald \& LAMBRECHTS, Win. (2013). Declarations for sustainability in high education: becoming better leaders, through addressing the university system. Journal Clean Production, Vol. 48, №. 1. Pp. 10-19. https://doi.org/10.1016/j.jclepro.2011.10.006 
46. LOZANO GARCÍA, Francisco José; KEVANY Kathleen; HUISING Donald. (2006) Sustainability in higher education: what is happening? Journal Clean Production, Vol. 14, No 9-11. Pp. 757-760. https://doi.org/10.1016/j.jclepro.2005.12.006

47. MÉJICO. CONACYT. (2017). Informe general del estado de la ciencia, la tecnología y la innovación, México 2017. Consejo Nacional de Ciencia y Tecnología. Disponible en Internet: http://www.siicyt.gob.mx/index.php/transparencia/informes-conacyt/ informe-general-del-estado-de-la-ciencia-tecnologia-e-innovacion/informegeneral-2017 Consultado: 10.12.2019.

48. MÉJICO. CONEVAL. (2018). Pobreza en Méjico. Disponible en Internet: https://www. coneval.org.mx/Medicion/Paginas/Pobreza_2008-2016.aspx Consultado: 17.06.2019.

49. MÉJICO. INCAE (2016). Emprendimiento juvenil en Méjico, Informe Nacional. Disponible en Internet: https://www.incae.edu > files > reporte_nacional_-_mexico_ final_corregido. Consultado: 29.11.2019.

50. MÉJICO. Instituto Nacional del Emprendedor. INE (2016). Diagnóstico 2016, Fondo Nacional del Emprendedor. Secretaria de Economía, Distrito Federal. Disponible en Internet: https://www.inadem.gob.mx/wp-content/uploads/2017/02/Diagnóstico_ FNE-2016.pdf Consultado: 22.01.2019.

51. MÉJICO. Instituto Nacional de Estadística y Geografía. INEGI (2015). Resumen de los resultados definitivos de los censos económicos 2014. Disponible en Internet: https://www.inegi.org.mx/contenidos/programas/ce/2014/doc/frrdf_ce2014.pdf Consultado: 02.07.2016.

52. MÉJICO. Instituto Nacional de Estadística y Geografía. INEGI (2018a). Encuesta intercensal 2015. Disponible en Internet: http://www.beta.inegi.org.mx/temas/ estructura/ Consultado: 18.03.2019.

53. MÉJICO. Instituto Nacional de Estadística y Geografía. INEGI (2018b). Encuesta Nacional de Ocupación y Empleo (ENOE). Disponible en Internet: http://www.beta. inegi.org.mx/temas/empleo/ Consultado: 05.03.2019.

54. MÉJICO. Observatorio Nacional del Emprendedor (2015). Construcción, generación y análisis de indicadores para medir el estado y evolución del ecosistema emprendedor en México. $1^{\text {a }}$ ed. Ciudad de Méjico: Secretaría de Economía, Diseño, Evaluación y Análisis de Políticas Públicas/Programa de las Naciones Unidas para el Desarrollo. 103 pp.

55. MOWERY, David C. (2005). The Bayh-Dole Act and High-Technology Entrepreneurship in U.S. Universities: Chicken, Egg, or Something Else (pp. 39-68). En: LIBECAP, Gary, University Entrepreneurship and Technology Transfer: Process, Design and Intellectual Property. Amsterdam: Elsevier.

56. O'NEIL, Gerald, HERSHAUER, James \& GOLDEN, Jay. (2009). The cultural context of sustainability entrepreneurship. Green Management International, Vol. 55, № 1. Pp. 33-46. https://doi.org/10.9774/GLEAF.3062.2006.au.00005

57. OCDE (2016). Perspectivas económicas de América Latina 2017: Juventud, competencias y emprendimiento. $1^{\text {a }}$ ed. Paris: OECD Publishing. 335 pp.

58. OCDE (2018). Employment Outlook 2018, OECD, 2018. Disponible en Internet: http://www. oecd.org/els/emp/oecd- employment-outlook-19991266.htm. Consultado: 23.01.2019.

59. ONU (2018). Informe de los Objetivos de Desarrollo Sostenible 2018. Nueva York. ONU. 
60. PÉREZ, María del Pilar y CALDERÓN, María Guadalupe. (2019). Avances normativos en la creación de empresas spin off universitarias en México. Entreciencias, Vol. 7, № 20, Pp. 53-64. https://doi.org/10.22201/enesl.20078064e.2019.20.68321

61. PERKMANN, Markus, TARTARI, Valentina, McKELVEY, Maureen, AUTIO, Erkko, BROSTRÖM, Anders, D'ESTE, Pablo, FINI, Ricardo, GEUNA, Aldo, GRIMALDI, Rosa, HUGHES, Alan, KRABEL, Stefan, KITSON, Michael, LLERENA, Patrick, LISSONI, Franceso, SALTER, Ammon \& SOBRERO, Maurizio (2013). Academic Engagement and Commercialisation: A Review of Literature on University-Industry Relations. Research Policy, Vol. 42, № 2. Pp. 423-442. https://doi.org/10.1016/j.respol.2012.09.007

62. RAMOS, Tomás, CAEIRO Sandra, VAN HOOF Bart, LOZANO Rodrigo, HUISING, Donald \& CEULEMAS, Kim. (2015). Experiences from the implementation of sustainable development in higher education institutions: environmental Management for sustainable Universities. Journal Clean Production, Vol. 106. № 1. Pp. 3-10. https://doi. org/10.1016/j.jclepro.2015.05.110

63. REDEMPRENDIA (2014). Análisis de las actividades de Investigación + Desarrollo + innovación + emprendimiento en Universidades de Iberoamérica. $1^{\text {a }}$ ed. Madrid: Netbiblio. $166 \mathrm{Pp}$.

64. RITALA, Paavo. (2019). Innovation for Sustentability: Sceptical, Pragmatic, and Idealist Perspectives on the Role of Business as a Driver for Change (pp. 21-34). En: BOCKEN, Nancy, RITALA, Paavo, ALBAREDA, Laura \& VERBURG, Robert (Edits.), Innovation for Sustainability. Suiza: Palgrave Macmillan.

65. SCHEYVENS, Regina, BANKS, Glenn \& HUGHES, Emma. (2016). The Private Sector and the SDGs: The Need to Move Beyond 'Business as Usual'. Sustainable Development, Vol. 24. № 1. Pp. 371-382. https://doi.org/10.1002/sd.1623

66. SCHOAR, Antoinette. (2010) The divide between subsistence and transformational entrepreneurship. Innovation Policy and the Economy, Vol. 10, № 1. Pp. 57-81. https:// doi.org/10.1086/605853

67. SCHUMPETER, Joseph Alois. (1947). The Creative Response in Economic History. The Journal of Economic History, Vol. 7, № 2. Pp. 149-159.

68. SHANE, Scott \& VENKATARAMAN, S. (2000). The promise of entrepreneurship as a field of research (pp. 171-184). En: CUERVO, Alvaro, RIBEIRO, Domingo \& ROIG, Salvador (Edits.), Entrepreneurship: Concepts, theory and perspective. Berlin Heidelberg: Springer-Verlag.

69. SILVESTRE, Bruno. (2015). A hard nut to crack! Implementing supply chain sustainability in an emerging economy. Journal Clean Production, Vol. 96, № 1. Pp 171-181. https:// doi.org/10.1016/j.jclepro.2014.01.009

70. SMILOR, Raymond \& SEXTON, Ronald. (1987). The Art and Science of Entrepreneurship. $1^{\text {a }}$ ed. Cambridge: Ballinger Pub. Co. 422 pp.

71. VAN LOOY, Bart, LANDONI, Paolo, CALLAERT, Julie, VAN POTTELSBERGHE, Bruno, SAPSALIS, Eleftherios \& DEBACKERE, Koenraad. (2011). Entrepreneurial Effectiveness of European Universities: An Empirical Assessment of Antecedents and Trade-Offs. Research Policy, Vol. 40, № 4. Pp. 553-564. https://doi.org/10.1016/j.respol.2011.02.001

72. VIVARELLI, Marco. (2013). Is entrepreneurship necessarily good? Microeconomic evidence from developed and developing countries. Industrial and Corporate change, Vol. 22, No 6. Pp. 1453-1495. https://doi.org/10.1093/icc/dtt005 
73. VON GEIBLER, Justus, PIWOWAR, Julius \& GREVEN, Annika. (2019). The SDG-Check: Guiding Open Innovation towards Sustainable Development Goals. Technology Innovation Management Review, Vol. 9, № 3. Pp. 20-37. https://doi.org/10.22215/ timreview/1222

74. WCED (World Commission on Environment and Development). (1987). Our common future. $1^{\mathrm{a}}$ ed. Oxford: Oxford university Press. $300 \mathrm{pp}$.

75. WENNEKERS, Sander, VAN WENNEKERS, André, THURIK, Roy \& REYNOLDS, Paul. (2005). Nascent entrepreneurship and the level of economic development. Small Business Economics, Vol. 24, № 3. Pp. 293-309. https://doi.org/10.1007/s11187-005-1994-8

76. WIPO (2011). 2011 World Intellectual Property Report. The Changing Face of Innovation. $1^{\text {a }}$ ed. Ginebra: World Intellectual Property Organization. 184 pp.

77. WRIGHT, Mike, BIRLEY, Sue \& MOSEY, Simon. (2004). Entrepreneurship and University Technology Transfer. Journal of Technology Transfer, Vol. 29, № 3-4. Pp. 235-246. https://doi.org/10.1023/B:JOTT.0000034121.02507.f3

78. WRIGHT, Mike, CLARYSSE, Bart, LOCKETT, Andy \& KNOCKAERT, Mirjam. (2008). Mid-Range Universities' Linkages with Industry: Knowledge Types and Role of Intermediaries. Research Policy, Vol. 37, № 8. Pp.1205-1223. https://doi.org/10.1016/j. respol.2008.04.021

79. YORK, Jeffrey G. y VENKATARAMAN, Sankaran. (2010). The entrepreneur-environment nexus: uncertainty, innovation and allocation. Journal Business Venture, Vol. 25, № 5. Pp. 449-463. https://doi.org/10.1016/j.jbusvent.2009.07.007

80. ZIELINSKI, Tymon, SAGAN, Iwona \& SUROSZ, Waldemar. (2018). Interdisciplinary Approaches for Sustainable Development Goals. $1^{\text {a }}$ ed. Suiza: Springer International Publishing AG. 245 pp.

Para citar este artículo:
PÉREZ-HERNÁNDEZ, Pilar y GRANILLO, Daniel Akenaton. (2020). Desafíos del emprendimiento universitario frente a los Objetivos de Desarrollo Sostenible en Méjico. Teuken Bidikay, Vol. 11, № 16. Medellín: Politécnico Colombiano. Pp 153-174. doi: 10.33571/teuken.v11n16a7

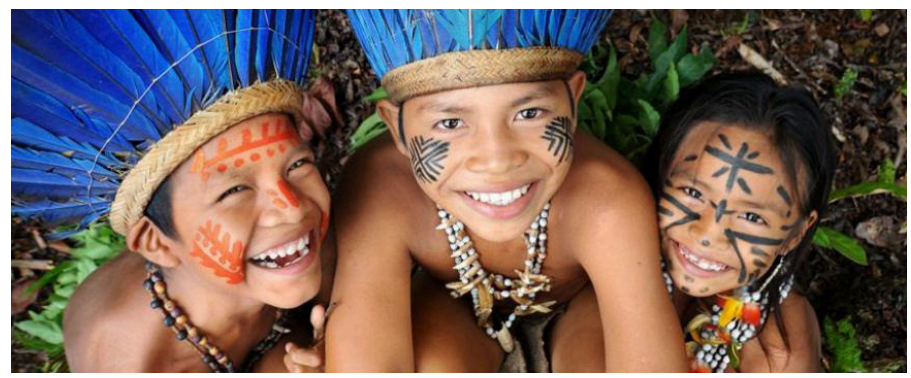

